\title{
Possible solution of the quarkonium polarization problem
}

\author{
S. P. Baranov* \\ P.N. Lebedev Institute of Physics, Lenin Avenue 53, Moscow 119991, Russia
}

(Received 10 November 2015; published 29 March 2016)

\begin{abstract}
We address the problem of $J / \psi$ spin alignment in hadron-hadron collisions and propose a natural solution to it using an extended nonrelativistic QCD model where the emission of soft gluons is treated as a series of explicit color-electric dipole transitions.
\end{abstract}

DOI: 10.1103/PhysRevD.93.054037

\section{INTRODUCTION}

Since first observed, quarkonium states remain a subject of intense studies on both theoretical and experimental sides. Quarkonium production in high-energy hadronic collisions serves as a complex probe of the hadron structure (parton model), perturbative QCD, and the formation mechanism of heavy-quark bound states. The latter seems to be the most tricky ingredient in the theory.

Several theoretical approaches are competing on the market, such as the color-singlet model and color-octet model. Each of them can be extended to next-to-leading order (NLO) or even next-to-next-to-leading order in their perturbative part, and each of them can be incorporated with collinear or $k_{T}$ factorization. None of the existing approaches is able to accommodate the whole set of experimental observables, and the worst problem is seen in the polarization. The overall situation is understood as a deep crisis. A review talk [1] given at the International Workshop on Heavy Quarkonium at CERN was concluded by stating that "NLO calculations either fail to make a polarization prediction or make predictions that disagree with the data."

The ordinary color-singlet model $[2,3]$ fails to describe the $p_{t}$ spectra of $J / \psi$ mesons. Going to higher orders in perturbative QCD (pQCD) can improve the situation but still does not make the whole story. We are interested in the bound states where the quarks are close in space. In such a case, long-wave gluons cannot resolve a composite system into its constituents. Thus, we have to leave $\mathrm{pQCD}$ at some resolution scale and switch to a different theoretical language, allowing that the gluons be emitted by the entire $Q \bar{Q}$ system and not by individual quarks.

The adequate language is provided by an approach called nonrelativistic QCD (NRQCD) factorization [4]. It makes use of an effective field theory $[5,6]$ to exploit the large mass of the heavy-quark $\mathrm{Q}$ to establish the factorization formula. This is another kind of perturbation theory where the small parameter is the size of the radiating system (or the quark relative velocity). In its mathematical aspects,

*baranov@sci.lebedev.ru
NRQCD bears strong resemblance to the classical multipole radiation theory.

In all practical applications with NRQCD, the final-state gluons changing the color and other quantum numbers of the $Q \bar{Q}$ pair and bringing this pair to the color-singlet state are regarded as carrying no energy momentum. This is in obvious contradiction with confinement, which prohibits the emission of infinitely soft colored quanta. In fact, the heavy-quark system must undergo a kind of final-state interaction where the energy-momentum exchange must be larger than at least the typical confinement scale (say, $\left.\Lambda_{\mathrm{QCD}}\right)$. This issue is not the matter of only kinematic corrections; without having a nonzero energy-momentum transfer, we cannot organize a transition amplitude with correct spin properties.

The role of final-state interactions was already noticed many years ago $[7,8]$ and even incorporated with NRQCD formalism [9]; however, no numerical predictions were made, and no attention was paid to polarization observables. In our previous paper [10], we studied the kinematical effects of final-state interactions in an explicit model considering the emission of soft gluons as a real radiation process. Here, we wish to extend our approach to $J / \psi$ polarization.

\section{MODEL}

The approach which we propose here may be regarded as a modification of the NRQCD scheme. At the first step, we consider the production of a heavy-quark pair in a shortdistance gluon-gluon fusion process. This is a purely perturbative step. All calculations are done in accordance with the standard QCD Feynman rules. The only not so usual issue is that we employ the $k_{t}$-factorization approach [11]. We see the advantages of the latter in the ease of including higher-order radiative corrections, which are taken into account in the form of unintegrated ( $k_{t}$-dependent) gluon densities. Unintegrated gluon densities also absorb the effects of soft gluon resummation; this regularizes infrared divergences and makes our theory applicable to even the small $p_{t}$ region. The details of calculations can be found in many of our previous publications $[12,13]$. To be precise, we only mention here that the present calculations were carried out with A0 gluon densities from Ref. [14], and the 

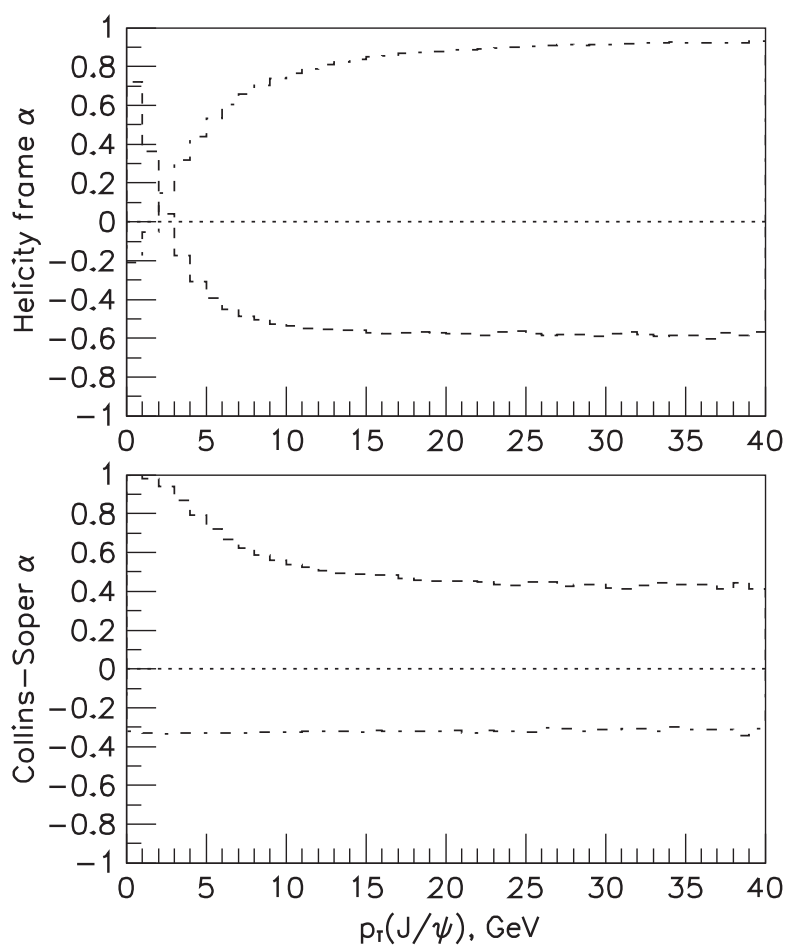

FIG. 1. Behavior of the polarization parameter $\alpha$ measured in the helicity frame (upper panel) and Collins-Soper frame (lower panel), as function of the $J / \psi$ transverse momentum. Notation of the curves: dashed, $J / \psi$ mesons coming from ${ }^{3} P_{2}^{[8]}$ states; dashdotted, from ${ }^{3} P_{1}^{[8]}$ states; dotted, from ${ }^{3} P_{0}^{[8]}$ or ${ }^{1} S_{0}^{[8]}$ states. All calculations are made in the $k_{t}$-factorization approach with $\mathrm{A} 0$ [14] gluon densities.

factorization and renormalization scales were set to $\mu_{R}^{2}=$ $\mu_{F}^{2}=m(Q \bar{Q})^{2}+p_{T}(Q \bar{Q})^{2}$, where the mass of the $Q \bar{Q}$ pair was taken slightly above the nominal $J / \psi$ mass in order to provide some phase space for the subsequent color-radiative transitions. For illustrative purposes, we rather arbitrarily used $m(Q \bar{Q})=m\left(\psi^{\prime}\right)=3.7 \mathrm{GeV}$ and set $\sqrt{s}=7 \mathrm{TeV}$. In fact, as we will see later, the results are insensitive to the choice of $m(Q \bar{Q})$. All information about the polarization is kept at this step in the $Q \bar{Q}$ spin density matrix.

If the quark pair represents a color-singlet state, then the quarkonium is complete, and it decouples from the rest of the system. If the quark pair represents a color-octet state, it further radiates nonperturbative long-wave gluons. We describe this step in terms of electric dipole (E1) transitions that dominante the multipole expansion. This makes the new point, because in the conventional NRQCD the emission of soft gluons was only declared but never considered as an explicit decay process.

In the case of ${ }^{3} P_{J}$ color-octet states, only a single E1 transition is needed to transform them into real mesons, ${ }^{3} P_{J}{ }^{[8]} \rightarrow J / \psi+g$. The structure of the relevant amplitudes is taken the same as for radiative decays of $\chi_{c}$ mesons $[15,16]$,
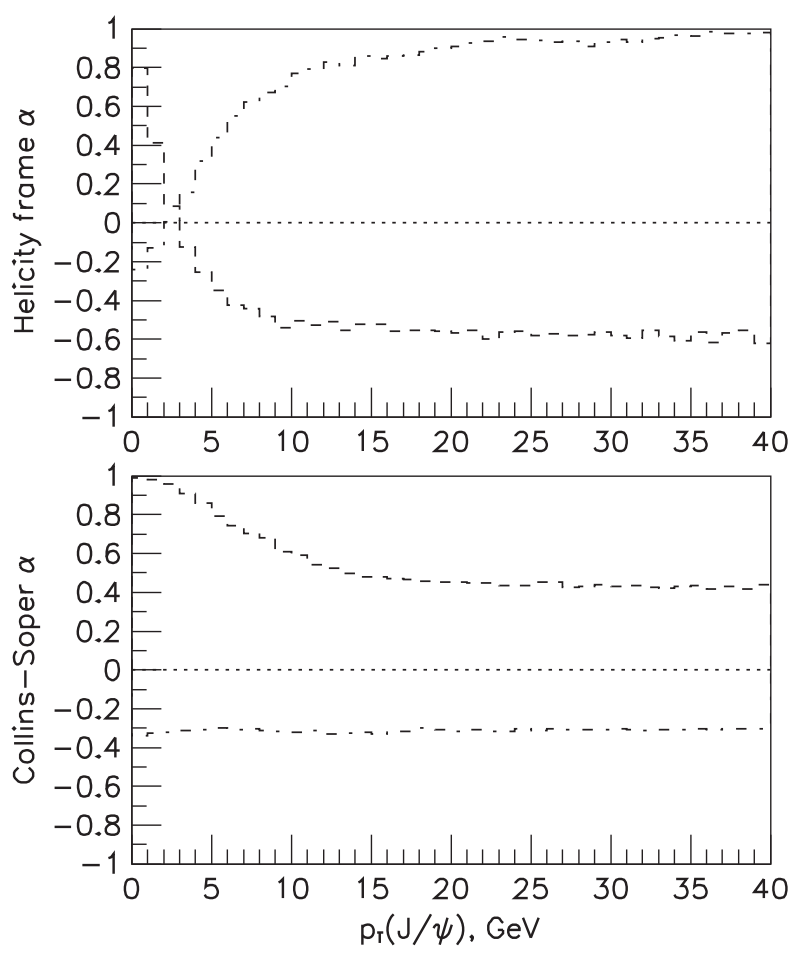

FIG. 2. Same as Fig. 1 but for leading-order collinear calculations with MSTW [17] gluon densities.

$$
\begin{aligned}
& \mathcal{A}\left(\chi_{c 0}(p) \rightarrow J / \psi(p-k)+\gamma(k)\right) \propto k_{\mu} p^{\mu} \varepsilon_{(J / \psi)}^{\nu} \varepsilon_{\nu}^{(\gamma)} \\
& \mathcal{A}\left(\chi_{c 1}(p) \rightarrow J / \psi(p-k)+\gamma(k)\right) \propto \epsilon^{\mu \nu \alpha \beta} k_{\mu} \varepsilon_{\nu}^{\left(\chi_{c 1}\right)} \varepsilon_{\alpha}^{(J / \psi)} \varepsilon_{\beta}^{(\gamma)}
\end{aligned}
$$

$$
\begin{aligned}
\mathcal{A}\left(\chi_{c 2}(p) \rightarrow\right. & J / \psi(p-k)+\gamma(k)) \\
& \propto p^{\mu} \varepsilon_{\left(\chi_{c 2}\right)}^{\alpha \beta} \varepsilon_{\alpha}^{(J / \psi)}\left[k_{\mu} \varepsilon_{\beta}^{(\gamma)}-k_{\beta} \varepsilon_{\mu}^{(\gamma)}\right],
\end{aligned}
$$

the only difference being in the overall normalizing factor. No matter whether the transition is perturbative or not, the only possible color structure for the amplitude of the process ${ }^{3} P_{J}^{[a]} \rightarrow J / \psi+g^{[b]}$ is $\delta^{a b}$, where $a$ and $b$ indicate the octet colors of the involved fields.

The polarization of the outgoing $J / \psi$ is now calculable from Eqs. (1)-(3) without any ambiguity, and the results are displayed in Figs. 1 and 2 for the $k_{t}$ and collinear factorization schemes with A0 [14] and MSTW-2008 [17] gluon densities, respectively. Shown there is the behavior of the parameter $\alpha$, which describes the angular distribution of the decay leptons with respect to a given axis: $d \sigma / d \theta \propto 1+\alpha \cos ^{2} \theta$. As one can see, the polarization of $J / \psi$ mesons coming from ${ }^{3} P_{2}{ }^{[8]}$ and ${ }^{3} P_{1}{ }^{[8]}$ states is opposite in sign so that these two contributions nearly compensate each other and bring the net polarization to zero. Needless to say, the spinless ${ }^{3} P_{0}{ }^{[8]}$ and ${ }^{1} S_{0}{ }^{[8]}$ states further dilute the polarization pattern. 

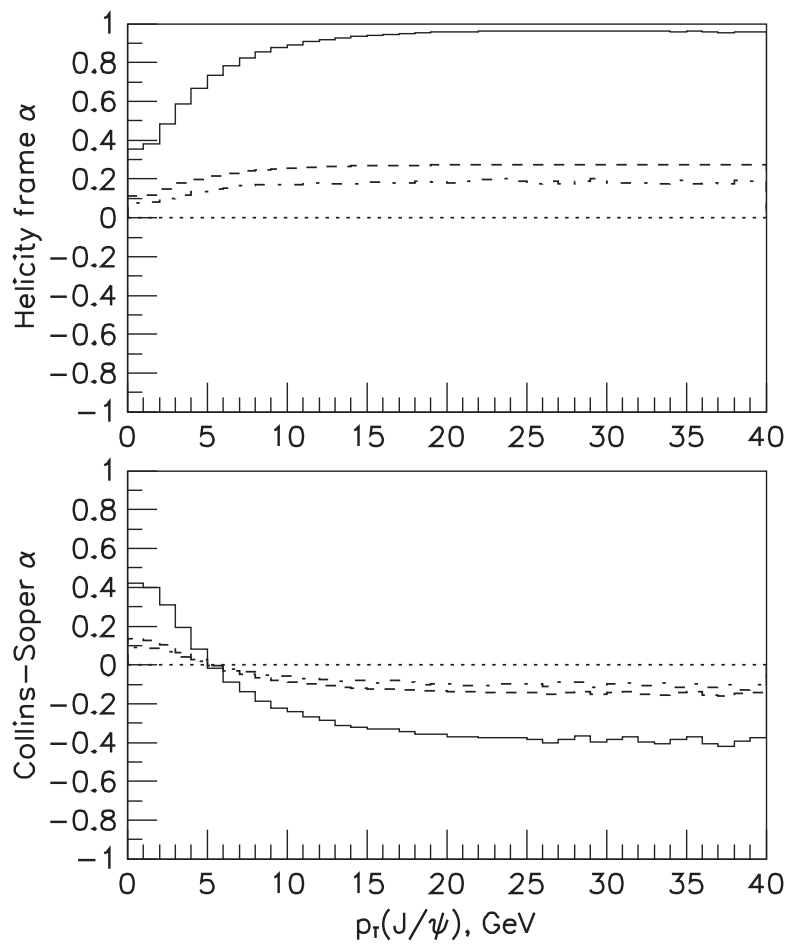

FIG. 3. Behavior of the polarization parameter $\alpha$ measured in the helicity frame (upper panel) and Collins-Soper frame (lower panel), as function of the $J / \psi$ transverse momentum. The original color-octet state is ${ }^{3} S_{1}$; different lines correspond to different intermediate states: ${ }^{3} P_{2}^{[8]}$ (dashed), ${ }^{3} P_{1}^{[8]}$ (dash-dotted), and ${ }^{3} P_{0}^{[8]}$ (dotted). The solid line shows the polarization of the originally produced ${ }^{3} S_{1}^{[8]}$ color-octet state.

If we start with the ${ }^{3} S_{1}$ color-octet state, we treat its transformation into real $J / \psi$ as two successive colorelectric dipole transitions ${ }^{3} S_{1}{ }^{[8]} \rightarrow{ }^{3} P_{J}{ }^{[8]}+g,{ }^{3} P_{J}{ }^{[8]} \rightarrow$ $J / \psi+g$ proceeding via either of the three intermediate states: ${ }^{3} P_{0}{ }^{[8]},{ }^{3} P_{1}{ }^{[8]}$, or ${ }^{3} P_{2}{ }^{[8]}$. Here, we exploit the same effective coupling vertices as above (1)-(3) and only change the sign of the radiated momentum $k$ for the "backward" case, ${ }^{3} S_{1} \rightarrow{ }^{3} P_{J}$. To describe the color structure of the process ${ }^{3} S_{1}^{[a]} \rightarrow{ }^{3} P_{J}^{[b]}+g^{[c]} \rightarrow J / \psi+g^{[b]}+g^{[c]}$, one could consider $f^{a b c}$ and $d^{a b c}$. Among these, $f^{a b c}$ is incompatible with the charge parity conservation, and that prohibits non-Abelian coupling between the final-state gluons. In the absence of non-Abelian interactions, the emission of gluons is fully similar to the emission of photons.

Our numerical results for the pure (noninterfering) ${ }^{3} P_{J}^{[8]}$ channels are displayed in Fig. 3. For comparison, we also present the parameter $\alpha$ as if $J / \psi$ would preserve the polarization of the ${ }^{3} S_{1}{ }^{[8]}$ state. As one can see, its original strong transverse polarization degrades substantially after two gluon emissions.

To estimate the interference effects between the different channels, we need to know the transition amplitudes
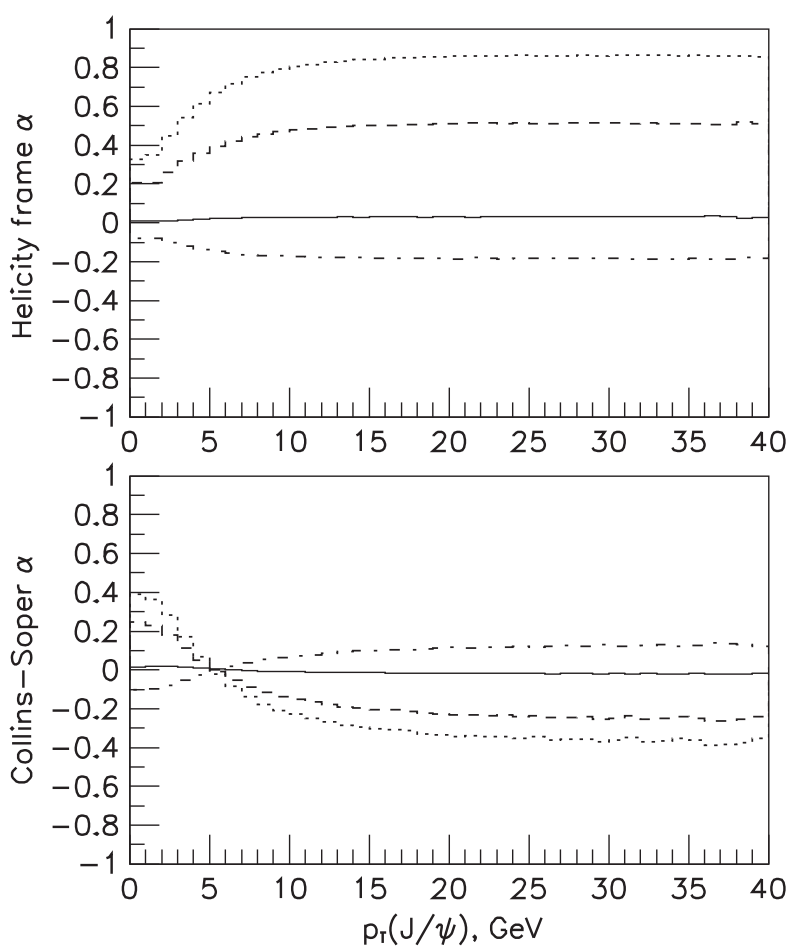

FIG. 4. $J / \psi$ polarization in the interference channels: $\left|{ }^{3} P_{0}^{[8]}-{ }^{3} P_{1}^{[8]}+{ }^{3} P_{2}^{[8]}\right|^{2}$ (dotted), $\left|{ }^{3} P_{0}^{[8]}+{ }^{3} P_{1}^{[8]}-{ }^{3} P_{2}^{[8]}\right|^{2}$ (dashed), $\left|{ }^{3} P_{0}^{[8]}-{ }^{3} P_{1}^{[8]}-{ }^{3} P_{2}^{[8]}\right|^{2}$ (dash-dotted), and $\left|{ }^{3} P_{0}^{[8]}+{ }^{3} P_{1}^{[8]}+{ }^{3} P_{2}^{[8]}\right|^{2}$

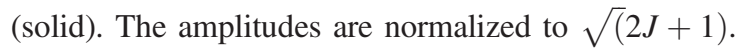

together with their relative phases. For the lack of $a$ priori information, we can only present here some extreme particular cases, such as neglecting some amplitudes or taking them with equal or opposite signs. We see that the interference can sometimes produce large polarization, as is shown in Fig. 4. At the same time, an assumption supported by heavy-quark effective theory that the amplitudes are proportional to $\sqrt{(2 J+1)}$ and have the same phase leads to nearly zero polarization (solid curve in Fig. 4). A similar behavior is observed in collinear calculations (not shown for saving space).

The final quarkonium polarization is insensitive to the choice of $m(Q \bar{Q})$, the mass of the decaying color-octet state. This fact is easily understandable. The transition amplitudes Eqs. (1)-(3) are proportional to the momentum of the emitted quantum so that by changing $m(Q \bar{Q})$ we only change the normalization of the amplitudes but not their structure. Once the transition has occurred, the resulting polarization is always the same. However, if the emitted momentum is set to zero, the amplitudes vanish, and we are driven to the unphysical conclusion that the original color-octet polarization is conserved.

So, irrespective of the original color-octet $Q \bar{Q}$ state, the polarization of the final state $J / \psi$ turns out to be weak; there is either a cancellation between the ${ }^{3} P_{1}{ }^{[8]}$ and ${ }^{3} P_{2}{ }^{[8]}$ 
contributions or depolarization of the ${ }^{3} S_{1}{ }^{[8]}$ state due to soft gluon radiation. So, the absence of strong $J / \psi$ polarization seems to be a natural and rather general property not connected to parameter tuning. Our approach has already demonstrated its virtue in describing the recent LHC data on the production and polarization of $\psi(2 S)$ mesons [18]. The work on the production and polarization of $J / \psi$ mesons is in progress.

Prior to going to the conclusions, we wish to make two additional remarks. Taken solely, the plots of Fig. 3 may correspond to an interesting scenario where the intermediate color-octet states are real on-shell bound states with definite mass and finite lifetime. This makes them similar to the ordinary $\chi_{c J}$ mesons. Such a scenario may have certain theoretical advantages. One can then try to solve Schrödinger's equation in some potential model (using a color-octet binding force) and evaluate the relevant color-octet wave functions by a direct computation. The nonrelativistic color transition probabilities would then become calculable physically meaningful quantities rather than merely fitting parameters as they were up to now.

Finally, we cannot exclude that in parallel with successive E1 transitions there present some other transition mechanisms. Recall, however, that we have [19] for the $\psi(2 S)$ branching fractions $\operatorname{Br}(\psi(2 S) \rightarrow J / \psi+X)$ : $\operatorname{Br}\left(\psi(2 S) \rightarrow \chi_{1}+\gamma\right): \operatorname{Br}\left(\psi(2 S) \rightarrow \chi_{2}+\gamma\right) \simeq 6: 1: 1$, while the first decay is strong and the other two are electromagnetic. Replacing $\alpha_{\mathrm{em}}$ with $\alpha_{s}$ in the latter cases would result in an enhancement factor of 30, thus making the E1 chain absolutely favorable. At least, the assumption of the dominance of two successive E1 transitions similar to the $\psi(2 S) \rightarrow \chi_{J} \rightarrow J / \psi$ chain does not look fully unreasonable.

\section{CONCLUSIONS}

We have considered the production of $J / \psi$ mesons in hadronic collisions using a model that interprets soft finalstate gluon radiation as a series of color-electric dipole transitions. We basically follow the NRQCD formalism but make further progress by representing the long-distance matrix elements in an explicit form inspired by the classical multipole radiation theory. This contrasts with the usual NRQCD calculations where the emitted gluons are never present explicitly in the final state and the spin structure of the transition amplitudes is not specified. At the same time, our treatment of gluon radiation is not the same as in the conventional color-singlet model at high-order perturbative QCD because our gluons are emitted by the entire $Q \bar{Q}$ system, and not by individual heavy quarks.

We find that the outgoing $J / \psi$ mesons appear to be not or only weakly polarized in all production channels, irrespective of the particular choice of nonperturbative color-octet matrix elements. Our study provides an easy and natural solution to a long-standing puzzle. We do not claim, however, that the proposed scenario is the only possible one; it is nothing but the first trial, one among a number of other acceptable approaches.

\section{ACKNOWLEDGMENTS}

The author conveys his thanks to Hannes Jung, Eugene Levin, Artem Lipatov, and Nikolai Zotov, who sadly passed away in January 2016, for their encouraging interest and useful discussions. This work was supported by the Deutsches Elektronen-Synchrotron (DESY, Federal Republic of Germany) Directorate in the framework of the Moscow-DESY project on Monte-Carlo implementations for HERA-LHC.
[1] G. Bodwin, https://indico.cern.ch/event/278195/.

[2] C.-H. Chang, Nucl. Phys. B172, 425 (1980); R. Baier and R. Rückl, Phys. Lett. 102B, 364 (1981); E. L. Berger and D. Jones, Phys. Rev. D 23, 1521 (1981).

[3] H. Krasemann, Z. Phys. C 1, 189 (1979); G. Guberina, J. Kühn, R. Peccei, and R. Rückl, Nucl. Phys. B174, 317 (1980).

[4] G. T. Bodwin, E. Braaten, and G. P. Lepage, Phys. Rev. D 51, 1125 (1995); 55, 5853(E) (1997).

[5] W. E. Caswell and G. P. Lepage, Phys. Lett. 167B, 437 (1986).

[6] B. A. Thacker and G. P. Lepage, Phys. Rev. D 43, 196 (1991).

[7] M. Beneke, Proceedings of Second Workshop on Continuous Advances in QCD, Minneapolis, MN, 1996, edited by M. Polikarpov (World Scientific, Singapore, 1996); arXiv: hep-ph/9605462.
[8] M. Mangano and A. Petrelli, Proceedings of the Quarkonium Physics Workshop, University of Illinois, Chicago, 1996 (unpublished); Report No. CERN-CH-TH-96-293;

[9] M. Beneke, I. Z. Rothstein, and M. B. Wise, Phys. Lett. B 408, 373 (1997).

[10] S. P. Baranov, Phys. Rev. D 91, 034011 (2015).

[11] L. V. Gribov, E. M. Levin, and M. G. Ryskin, Phys. Rep. 100, 1 (1983); E. M. Levin and M. G. Ryskin, Phys. Rep. 189, 268 (1990).

[12] S. P. Baranov, Phys. Rev. D 66, 114003 (2002).

[13] B. Andersson et al. (Small-x Collaboration), Eur. Phys. J. C 25, 77 (2002); J. R. Andersen et al. (Small-x Collaboration), Eur. Phys. J. C 35, 67 (2004); 48, 53 (2006).

[14] H. Jung, http://www.desy.de/ jung/cascade/updf.html.

[15] A. V. Batunin and S. R. Slabospitsky, Phys. Lett. B 188, 269 (1987). 
POSSIBLE SOLUTION OF THE QUARKONIUM ...

[16] P. Cho, M. Wise, and S. Trivedi, Phys. Rev. D 51, R2039 (1995).

[17] A. D. Martin, W. J. Stirling, R. S. Thorne, and G. Watt, Eur. Phys. J. C 63, 189 (2009).
PHYSICAL REVIEW D 93, 054037 (2016)

[18] S. P. Baranov, A. V. Lipatov, and N. P. Zotov, Eur. Phys. J. C 75, 455 (2015).

[19] K. A. Olive et al. (Particle Data Group), Chin. Phys. C 38, 090001 (2014). 